\section{BIOTECNOLOGÍA MARINA Y ACUICULTURA}

\author{
Antonio Figueras \\ Instituto de Investigaciones Marinas \\ Consejo Superior de Investigaciones Científicas \\ antoniofigueras@csic.es \\ Beatriz Novoa \\ Instituto de Investigaciones Marinas \\ Consejo Superior de Investigaciones Científicas \\ beatriznovoa@iim.csic.es
}

\section{MARINE BIOTECHNOLOGY AND AQUACULTURE}

Cómo citar este artículo/Citation: Figueras, A. y Novoa, B. (2014). "Biotecnología marina y acuicultura”. Arbor, 190 (768): a153. doi: http://dx.doi.org/10.3989/arbor.2014.768n4007

Recibido: 9 junio 2014. Aceptado: 14 julio 2014.

RESUMEN: Debido a la situación de los caladeros y al encarecimiento del proceso extractivo, el consumo de pescado y marisco se apoya cada vez más en la acuicultura. España es el tercer país consumidor de pescado del mundo, por detrás tan solo de Japón y Noruega. La biotecnología puede acelerar la consecución de estos objetivos. Sin embargo, no todo es producción de alimento; gracias a la biotecnología asociada al mundo acuático podemos generar combustible, energía, medicamentos y muchas otras aplicaciones que nos permiten valorar cada vez con más la riqueza del mar y su biodiversidad.

PALABRAS CLAVE: Biotecnología; acuicultura; pesca; medicina; farmacología; biodiesel; antitumorales; biopolímeros.
Copyright: (c) 2014 CSIC. Este es un artículo de acceso abierto distribuido bajo los términos de la licencia Creative Commons Attribution-Non Commercial (by-nc) Spain 3.0.

ABSTRACT: As a result of overfishing and the escalating cost of fishing, fisheries are unable to provide all the marine products that the world market demands. This shortfall is being met by aquaculture. After Japan and Norway, Spain is one of the world's leading consumers of fish and seafood. To reduce the losses from disease, increase growth and reproduction rates and juveniles survival... Biotechnology can help achieve these goals. However, there is more than food production in the use of Biotechnology in the marine world. Generation of biodiesel, medicines or new materials have their roots on the application of biotechnology to the marine world.

KEYWORDS: Biotechnology; aquaculture; fisheries; medicine; pharmacology; biodiesel; antitumorals; biopolymers. 


\section{INTRODUCCIÓN}

Los océanos constituyen más de las dos terceras partes de nuestro planeta. Lógicamente son fuente de muchos recursos, entre ellos alimento. Alrededor de un $35 \%$ de los productos pesqueros que se consumen en el mundo proceden de la acuicultura y este porcentaje sigue incrementándose paulatinamente. El 70\% de los caladeros internacionales se encuentra en estado de sobreexplotación y el nivel de capturas actual procedente de las actividades pesqueras ha llegado prácticamente al máximo que puede alcanzarse. Además, el continuo encarecimiento de la pesca debido a la imparable subida del precio del combustible hace que el futuro de esta actividad sea cada vez más complicado. La producción acuícola española la lidera Galicia, con una producción anual cercana a las 210.000 toneladas, y que constituye la mayor parte de la producción total de la acuicultura española, cifrada en torno a las 270.000 toneladas. La biotecnología podría ayudar a conseguir una producción económicamente rentable de animales sanos con un impacto ambiental limitado.

La biotecnología consiste en aprovechar todo lo que tienen los seres vivos en nuestro beneficio y no solo en la producción de animales para el consumo. Por esto, la biotecnología marina incluye además de la producción de alimento mediante el cultivo de organismos marinos, el aprovechamiento de los recursos de nuestros mares como fuente de productos naturales o incluso como energías alternativas.

Los océanos contienen una importante biodiversidad todavía por descubrir. En los últimos años los progresos en nuevas técnicas moleculares y distintas tecnologías marinas han permitido por ejemplo un considerable aumento del número de patentes sobre genes de organismos marinos (Arrieta, ArnaudHaondy Duarte, 2010).

El científico japonés Shimomura, galardonado con el Nobel de Química en 2008, quedó fascinado por la química de la bioluminiscencia y entre las décadas de 1960 y 1970 recogió más de un millón de medusas en el estado de Washington. Dedicó los siguientes 40 años a examinar de forma meticulosa las proteínas encargadas del brillo y encontró una proteína, la aecuorina, que producía luz azul, la cual posteriormente era convertida en luz verde por la acción de una proteína fluorescente verde (green fluorescence protein o GFP). Con técnicas de biología molecular, al alcance de cualquier laboratorio, se puede unir el gen de la GFP al gen de la proteína que se desee, de tal forma que la célula que incorpore esta construcción expresará una proteína en la que se ha añadido la GFP a su secuencia original. Esto nos permite ver (y no es una figura poética sino la realidad) como una bacteria interactúa con el sistema inmune o comprobar si un tratamiento contra el cáncer es eficaz (Shimomura, 2009). Su aplicación en todos los laboratorios de biología molecular del mundo nos enseña las posibilidades que pueden llegar a tener organismos marinos todavía sin explotar.

A ver si el mar, además de servir de basurero y de despensa (mientras dure la pesca y la acuicultura), nos guarda sorpresas en la lucha contra el cáncer y otras enfermedades que tanto nos preocupan. Para esto sirve la ciencia.

\section{LA ACUICULTURA}

El término acuicultura se refiere a todas aquellas actividades cuyo fin es la producción de organismos acuáticos, ya sean de aguas dulces, salobres o saladas. Este proceso abarca todas las etapas del desarrollo, desde los primeros estadíos hasta los adultos. Hoy en día, algas, crustáceos, peces y moluscos constituyen los grandes grupos de interés acuícola.

La idea de "cultivar el mar" no es nueva. En Hawai se descubrieron restos prehistóricos de estanques que fueron utilizados para la estabulación y mantenimiento de animales acuáticos. Ya en el año 1.400 a.C. se conoce que existían leyes para proteger a los piscicultores de los ladrones furtivos en la región Indo-Pacífica (Iversen, 1976). El primer tratado sobre acuicultura se remonta al año 475 a.C. cuando Fan-Li, en China, escribe el libro "Cría de peces" donde se recogen las indicaciones necesarias para el cultivo de la carpa común, Ciprinus carpio (Milne, 1972). En el siglo IV a.C., Aristóteles menciona el cultivo de ostras en Grecia, mientras que Plinio da detalles del mismo en Roma. En Galicia existen registros para poder asegurar que el consumo de moluscos bivalvos se remonta al siglo IV a.C. ya que han aparecido restos de conchas de ostras, mejillones y almejas, en depósitos denominados "concheiros" en los alrededores de lo que en su día fueron asentamientos humanos (Figueras, 2007).

Parece razonable pensar que el consumo de los productos de la pesca tiene que fundamentarse cada vez más en la acuicultura, lo que confirma las altas expectativas de crecimiento para las producciones acuícolas en un futuro próximo. España es el tercer país consumidor de pescado del mundo con 35 kilogramos por persona y año, por detrás tan solo de Japón (45 kilo- 
gramos por persona y año) y Noruega (40 kilogramos por persona y año).

La acuicultura es una actividad de creciente importancia para la Unión Europea y para España debido, entre otras razones, al impacto de esta actividad sobre el empleo en comunidades litorales. Según datos de la FAO (http://www.fao.org/fishery/statistics/es), Europa es el segundo continente en producción acuícola, caracterizándose las especies que se producen por su elevado valor económico. España está situada en el puesto 14 de la clasificación mundial. Su acuicultura marina supera las 300.000 toneladas (que se reducen a 53.900 si se excluye el mejillón), lo que supone el $24 \%$ de la producción pesquera española.

En 2011 la producción mundial de peces en acuicultura superó por primera vez a la de vacuno. La diferencia aumentó en 2012 con una producción de peces de acuicultura de 66 millones de toneladas mientras que se produjeron 63 millones de toneladas de carne de vacuno. Además, probablemente ya se esté consumiendo más pescado producido en granjas acuáticas que capturado por los métodos tradicionales de pesca.

Para conseguir la producción económicamente rentable de animales sanos con un impacto ambiental limitado es necesario mejorar las tasas de crecimiento y la eficacia de la alimentación y reproducción, disminuyendo las pérdidas causadas por las enfermedades, mediante la mejora la respuesta inmune, las técnicas de diagnóstico y las medidas profilácticas. La biotecnología puede acelerar la consecución de estos objetivos.

Es interesante señalar que en el desarrollo de la biotecnología aplicada a especies acuícolas, España todavía no ha perdido el tren, entre otras causas porque esta área está en su inicio en los países avanzados de nuestro entorno (Estados Unidos, Canadá y Noruega). Además, existen en nuestro país grupos de investigación punteros trabajando en aspectos biotecnológicos aplicados a la acuicultura, como es la búsqueda de caracteres genéticos (relativos a la reproducción, el crecimiento y la resistencia a las enfermedades), para mejorar, sin descartar la manipulación genética, la producción de las especies.

\section{RECURSOS GENÓMICOS DE ESPECIES DE INTERÉS COMERCIAL EN ACUICULTURA}

Los transcriptomas, son la principal fuente de datos genómicos en organismos no modelo. La obtención de colecciones de genes que se están expresando o "ESTs" (Expressed Sequence Tags) suele constituir el inicio de la obtención de información genómica.
Conocer qué genes se expresan en una determinada condición supone un primer paso para profundizar en la biología de esa especie y analizar los factores que afectan a su crecimiento, reproducción, respuesta inmune, etc. Estas secuencias se suelen emplear en el diseño de microarrays que se utilizan en experimentos de determinación de niveles de expresión génica. Existen bases de datos de ESTs para especies como la ostra japonesa (C. gigas), GigasBase de ostra en Francia (http://www.ifremer.fr/GigasBase), y el mejillón Mytilus galloprovincialis (Mytibase) o la base de datos Marine Genomics (www.marinegenomics.com) (Figueras, Costa y Novoa, 2012). En el caso de los peces, el incremento de las bases de datos de ESTs se ha hecho más acusado en los últimos años. Los salmónidos, son los peces en los que más se ha avanzado en la aplicación de herramientas de genómica. Un consorcio de grupos de Canadá, Francia, Noruega y Estados Unidos obtuvieron más de 300.000 secuencias de cDNA de salmónidos que se organizaron en 40.000 contigs únicos con los que se construyó un microarray que comprende 16.006 genes y que se ha empleado para detectar patrones de expresión génica frente a infecciones experimentales y a cambios ambientales y fisiológicos a pequeña escala (Rise et al., 2004). Sin embargo, queda mucho por recorrer. Es importante señalar que mientras que para trucha arco iris el número de secuencias depositadas en el GeneBank es de 244.984 y para salmón Atlántico es de 236.009, solo hay unas 200 secuencias de rodaballo. La información genómica en dorada y lubina es más amplia existiendo cerca de 2.448 y 24.452 secuencias, respectivamente depositadas en GeneBank. El número de ESTs de pez cebra, especie de pez modelo, es de 833.880 .

Las nuevas técnicas de secuenciación masiva como el RNA-seq permiten la obtención de un gran número de secuencias expresadas con menor inversión en tiempo y dinero y por ello están sustituyendo a la obtención de ESTs/microarrays a la hora de realizar estudios de expresión génica.

El genoma es el conjunto del material hereditario de un organismo, en el que se encuentran las instrucciones para el desarrollo y funcionamiento del mismo y que son transmitidas de generación en generación, de padres a hijos. Aparte del interés básico, la comparación de genomas podría ayudar a descubrir marcadores genéticos que se utilizarán en procesos de selección de estirpes con características de interés como una alta tasa de crecimiento o resistencia a las enfermedades. 
En moluscos producidos para consumo, se ha secuenciado el genoma completo de la ostra japonesa (Crassostrea gigas) (Zhang et al., 2012). Entre otras razones se seleccionó porque su genoma está entre los más pequeños de los genomas de moluscos (500-600 $\mathrm{Mb}$ ) además de ser una de las especies de moluscos mejor estudiadas desde el punto de vista genético, lo que incluye la existencia de mapas de ligamiento, genotecas en vectores BAC, y una gran cantidad de información sobre secuencias genómicas codificantes. Recientemente se ha publicado el borrador del genoma de la ostra perlífera (Pinctadafucata) (Takeuchi et al., 2012). En la actualidad investigadores del CSIC y de la Universidad de Vigo están secuenciando el del mejillón en su versión gallega (Mytilus galloprovincialis), que se extiende hasta el País Vasco, Francia y el sur de Inglaterra y alcanza el Mediterráneo.

El genoma del mejillón podrá valer, entre otras cosas, para domesticar y mejorar este recurso, de momento una especie salvaje. Galicia es, después de China, la segunda productora mundial de mejillón, con 210.000 toneladas anuales, una facturación de 150 millones de euros, 11.500 puestos de trabajo directo y 7.000 indirectos. Esta producción constituye el $80 \%$ del total de la acuicultura marina de España.

Entre los genomas de peces, un consorcio internacional está secuenciando el del salmón atlántico (Salmo salar), la especie con una mayor producción comercial. Se pretende obtener un genoma que identificará un mapa de todos los genes del salmón atlántico y que servirá de referencia para los genomas de otros peces (Davidson et al., 2010).

Recientemente se ha publicado la secuenciación del genoma de un pez plano Cinoglossus sinensis (Chen et al., 2014).

Otras especies cuyos genomas se están secuenciado son: el pez gato Ictalurus punctatus (http://catfishgenome.org/cbarbel), el medaka, Oryzias latipes http://mbase.nig.ac.jp/mbase/medaka_top.html, un ciclído http://cichlid.umd.edu/CGCindex.html, el Fugu rubripes, http://www.fugu-sg.org, el Tetraodon nigroviridis, http://www.cns.fr/spip/Tetraodon-nigroviridis-a-fish-with.html, el Xiphophorus, http:// www.xiphophorus.txstate.edu/ y el Gasterosteus acculeatus, https://www.broadinstitute.org/models/stickleback. Además, se está finalizando la secuenciación del genoma del rodaballo (Scophtalmus maximus) por un consorcio español integrado por científicos del CSIC, del CRG y de la Universidad de Santiago de Compostela.
Tanto los genomas como los mapas de ligamiento se pueden utilizar para identificar los genes responsables de un carácter de interés para el cultivo. Normalmente, se trata de caracteres cuantitativos, determinados por varios genes en interacción con el ambiente. En acuicultura, como en otras actividades de producción animal, los caracteres de mayor interés incluyen la tasa de crecimiento o la resistencia a determinadas enfermedades. Los genes responsables de la variación cuantitativa se llaman "loci de caracteres cuantitativos", o QTL (quantitative trait loci). Se han descrito QTLs para resistencia frente a dos parásitos protozoos causantes de mortalidades en la ostra americana (Crassostrea virginica) (Figueras, Costa y Novoa, 2012). En peces, se han descrito QTLs en el salmón atlántico asociados a tasas de crecimiento en peso (Gutierrez et al., 2012), a resistencia a infecciones virales (Houston et al., 2008) o al color de la carne (Baranski, Moen y Våge, 2010). Estos QTLs se emplean en programas de selección asistida por marcadores marker assisted selection (MAS).

\section{ACUICULTURA Y TRANSGÉNICOS}

Para algunos la biotecnología son solo "los transgénicos". Un invento "diabólico" que consiste, en que, mediante técnicas ingeniosas y sencillas, conseguimos incrementar las copias de un gen en cada individuo. Así se ven reforzados algunos procesos biológicos necesarios para mejorar la producción.

La Organización para los alimentos y la agricultura de las Naciones Unidas predice que la producción de la acuicultura se doblará en la próxima década. Dado que la acuicultura en aguas costeras puede dañar los ecosistemas, extendiendo enfermedades de peces y moluscos, modificando hábitats, causando contaminación por el exceso de nutrientes y antibióticos y mediante la introducción de especies exóticas, se piensa que los legisladores podrían llegar a exigir que las granjas de peces se instalen solo en tierra. Si esto es así, los peces modificados genéticamente que crezcan rápido podrían ser la única salida para que esta industria sea económicamente competitiva.

La transgénesis se puede definir como la introducción de ADN en un genoma, de modo que se mantenga estable de forma hereditaria y afecte a todas las células en los organismos multicelulares.

Generalmente, en animales, el ADN extraño, llamado transgén, se introduce en zigotos, y los embriones que hayan integrado el ADN extraño en su genoma, previamente a la primera división, producirán un or- 
ganismo transgénico; de modo que, en algunos, el transgén pasará a las siguientes generaciones a través de la línea germinal (gametos).

En general existe rechazo en la población hacia los organismos transgénicos, probablemente como una reacción hacia lo desconocido. La ingeniería genética es una vía alternativa a la selección artificial dirigida (aplicada ya en algunos casos). Tecnologías como la manipulación del genoma de estos animales mediante la inserción de genes o la manipulación de los ya existentes permiten conseguir animales transgénicos de alta tasa de crecimiento o resistentes a enfermedades sin necesidad de selección genética, normalmente lenta y limitada.

No sabemos si los salmones transgénicos presentan algún tipo de riesgo real pero sí se intuyen riesgos potenciales:

- El salmón cultivado (transgénico o no) se escapa de las jaulas en las que se cría en el agua.

- El salmón modificado genéticamente puede cruzarse con los salvajes liberando sus genes de la hormona del crecimiento a las poblaciones salvajes con resultados impredecibles.

- Las metodologías de esterilización no son eficaces al $100 \%$ y existe una gran variación en los resultados entre grupos de animales.

- Los salmones modificados genéticamente comen tres veces más en el laboratorio que los no modificados pero es menos cuidadoso con sus depredadores.

- ¿Serían menos capaces de sobrevivir en la naturaleza?

Los avances logrados recientemente en la transferencia de genes han ofrecido la posibilidad de manipular el crecimiento en los peces a través de la inserción de los genes de la hormona del crecimiento. Desde que varias compañías presentaron su solicitud de permiso para comercializar salmón transgénico, no ha cesado de crecer la controversia en torno a estos salmones transgénicos. La investigación sobre líneas de peces transgénicas ha estado en marcha durante los últimos quince años en todo el mundo, incluyendo fundamentalmente el salmón del Pacífico (Onchorrhynchus kisutch), varios miembros de la familia de los salmónidos y otros peces de interés comercial como el pez gato o la tilapia. Este año 2014, acaba de superar el último obstáculo antes de llegar a los platos el salmón transgénico, conocido como AquaAdvantage Salmon, que desde hace 23 años desarrolla la empresa estadounidense Aquabounty. La Agencia de
Alimentación de EE UU (FDA) ha concluido que comerlo es seguro (Ledford, 2013). A este salmón se le ha introducido un gen que regula la producción de la hormona del crecimiento de una especie de salmón próxima. Además, se ha introducido un "interruptor genético" que le permitirá producir esta hormona también en invierno cuando las temperaturas bajas ralentizan su producción en los animales salvajes y por tanto su tasa de crecimiento.

El mayor problema de los transgénicos, como se apuntó anteriormente, no es tanto el que nos hagan daño al comerlos sino su posible impacto ecológico. Al ser "más eficaces" podrían competir de forma ventajosa con las poblaciones naturales y podrían llegar a eliminarlas. Por eso habría que cultivar los transgénicos en tanques situados en tierra para garantizar que no se escapen.

Obviamente se trata de un debate complejo con partidarios y detractores: ¿preferimos comer animales tratados con productos químicos o antibióticos, o animales que crecen más rápido porque gracias a la incorporación de más copias de sus propios genes no necesiten de "tratamientos"? Aunque esos productos químicos ya hayan desaparecido antes de comerlos, no podemos olvidar que al final van al mar y nos los acabamos encontrando.

\section{BIODIVERSIDAD Y BIOTECNOLOGÍA}

Según "Sustaining Life", un libro realizado por un centenar de expertos presentado en el Programa de Medio Ambiente de Naciones Unidas (Chivian y Bernstein, 2008), una nueva generación de medicamentos puede desaparecer como consecuencia de la pérdida de biodiversidad.

La naturaleza guarda miles de secretos para el desarrollo de nuevos tipos de analgésicos y métodos desconocidos para la recuperación de tejidos y órganos perdidos. En el mundo marino tenemos múltiples ejemplos de la utilidad de diversas especies para usos biomédicos u otras aplicaciones tecnológicas, de hecho, hoy en día existen ya casos de comercialización de distintas sustancias a partir de especies marinas. A modo de ejemplo podemos citar:

\section{Medicamentos}

La aniquilación de los tiburones significaría la desaparición de la escualamina, una sustancia con propiedades antibióticas y antifúngicas (Ghodbane et al., 2013). Además su empleo permite controlar el crecimiento de tumores cerebrales en ratas experimentales 
prolongando su vida. Esta sustancia ataca los tumores al inhibir la formación de nuevos vasos sanguíneos.

El caso más conocido en nuestro país de un medicamento derivado de especies marinas, en concreto de la ascidia Ecteinascidia turbinata es el de Yondelis ${ }^{\circledR}$ (trabectedina). Yondelis ${ }^{\circledR}$, producido por la empresa española PharmaMar, tiene un mecanismo de acción novedoso, resultante de su unión al ADN interfiriendo en los procesos de división celular, de transcripción genética y en los sistemas de reparación del ADN.

Yondelis ${ }^{\circledR}$ ha recibido la autorización de comercialización de la Comisión Europea para el tratamiento del sarcoma de tejido blando avanzado (STB) después de que hayan fracasado las antraciclinas y la ifosfamida, o en pacientes que no pueden recibir tratamiento con estos agentes. Además, Yondelis ${ }^{\circledR}$ se encuentra en ensayo clínico pivotal de fase III para cáncer de ovario. También se están llevando a cabo ensayos de fase II con Yondelis ${ }^{\circledR}$ para cáncer de mama y de próstata y de fase I para tumores pediátricos.

Esta empresa está trabajando además en otros interesantes compuestos: Aplidin ${ }^{\circledR}$ es un agente antitumoral de origen marino, aislado originalmente del tunicado marino Aplidium albicans (Barboza et al., 2012); Kahalalide F, un nuevo agente antitumoral de origen marino aislado del molusco marino Elysia rufescens (Salazar et al., 2013); ES-285 aminoalcohol antitumoral de origen marino aislado del molusco Mactromerispolynyma; Zalypsis ${ }^{\circledR}$ (PM00104/50) relacionado con el compuesto natural marino Jorumycina y con la familia de las Renieramycinas que se derivan de moluscos y esponjas (Moneo et al., 2014).

Los moluscos gasterópodos de la familia "Conidae" producen un compuesto con un efecto analgésico 1.000 veces superior al de la morfina, útil para aliviar el dolor a pacientes de sida y cáncer. La ziconotida, una forma sintética del veneno de un caracol de mar permite un alivio del dolor de moderado a completo en aproximadamente el $53 \%$ de los pacientes tratados (Kijjoa y Sawangwong, 2004).

En animales tan próximos a nosotros como son los mejillones, se ha descubierto una nueva clase de péptidos antimicrobianos, con actividades antivirales y antibacterianas, Ilamada myticina C (Balseiro et al., 2011). Los péptidos antimicrobianos (AMP) son pequeñas moléculas, presentes en la gran mayoría de organismos, que actúan como antibióticos naturales ante determinadas enfermedades. Forman parte del sistema inmunitario innato, que se encarga de defender al organismo de todo lo que este no reconoce como propio.

\section{"Pegamentos" marinos}

Es sorprendente la capacidad que tiene el mejillón para adherirse a cualquier superficie y para aguantar varias veces su peso. Los mejillones son capaces de viajar adheridos a cascos de barcos a gran velocidad y no caerse. Las proteínas adhesivas presentes en el pegamento natural que los mejillones secretan para adherirse a las rocas y entre sí llamó la atención de investigadores financiados por el National Institute of Health de Estados Unidos y la NASA. Su aplicación en cirugía permite que los tiempos de recuperación sean más pequeños, y que las cicatrices sean menos visibles. Aunque la sustancia se comercializa, su uso no ha llegado aún a la experimentación clínica. Aún es necesario averiguar la composición correcta para la aplicación en humanos por lo que algunos grupos científicos están buscando versiones sintéticas de ese pegamento.

Los pegamentos marinos tienen que mantener su eficacia y no disolverse en el agua. Los científicos mostraron que los mejillones hacen más fuerte su pegamento con moléculas de hierro. Sin embargo, se desconocen los detalles mecánicos. El pegamento está hecho de una mezcla de proteínas que pueden purificarse e incluso sintetizarse pero mucha de su fuerza adhesiva proviene de su estructura que se pierde en la purificación y de momento no se puede imitar artificialmente (Lee, Scherer y Messersmith, 2006).

Mientras no se descubre cómo replicar la estructura del pegamento del mejillón los investigadores dirigen su atención a un gusano que mediante su pegamento une partículas de arena para organizar un tubo que le sirve como casa (Wang y Stewart, 2012). En este caso, se unen dos materiales similares (arena-arena), mientras que el mejillón une su biso a una roca húmeda. La variedad en la composición de los pegamentos marinos es bastante elevada. Otros animales como los percebes o los cirrípedos no usan DOPA, que se considera central en el pegamento de los mejillones o de los gusanos tubícolas.

\section{Biocombustibles}

Numerosas empresas e iniciativas científicas están actualmente intentando desarrollar biocombustibles a partir de algas y otros microorganismos marinos fotosintéticos, como las cianobacterias. La idea es prometedora pero la viabilidad económica por ahora no es muy evidente. 
Las algas presentan numerosas ventajas sobre los biocombustibles (que algunos han pasado a llamar agrocombustibles): se reproducen solas, no "invaden" suelo agrícola y ni siquiera necesitan agua limpia o dulce para crecer (Vanegas y Bartlett, 2013). Según Exxon esto implica que no haría falta, en un futuro, adaptar las infraestructuras a este nuevo tipo de combustible (es decir, se ahorrarían costes añadidos), sino que "se procesaría en las mismas refinerías actuales y se suministraría en las mismas gasolineras". Actualmente, los biocombustibles agrícolas ya están presentes en el mercado como un producto mezclado con los combustibles tradicionales. España cuenta con algunas 'biogasolineras' que los suministran.

En cuanto a previsiones de futuro, la compañía petrolera estima que las algas podrían producir, potencialmente, más de 20.000 litros de combustible por hectárea y año (Soh et al., 2014). Los objetivos no son excesivamente ambiciosos, aunque sí realistas, similares al potencial de otras empresas. El problema es lograrlo.

Por otra parte, recientemente ha completado un vuelo el primer avión (un Boeing 737-800) propulsado con una mezcla de biodiesel derivado de algas y combustible. La aeronave, de la compañía Continental Airlines, completó un trayecto de 90 minutos por el golfo de México. La prueba ha sido la última de una serie de experimentos de la industria aeronáutica, que espera poder aplicar biocombustibles en cinco años.

\section{Biopolímeros}

Escribir que dependemos del petróleo es una perogrullada, una obviedad. También es cierto que tar- de o temprano esta fuente de energía se agotará y no solo como motor del mundo sino como solución para muchas de nuestras necesidades. Los polímeros sintéticos derivados del petróleo como polietileno o nylon están presentes en casi todos los productos de consumo. Estos plásticos necesitan muchos años para degradarse. Los problemas de eliminación y la falta de materia prima han llevado a los investigadores a inventar nuevas alternativas.

El quitosán es un biopolímero marino derivado de la quitina que se encuentra en el caparazón de algunos crustáceos marinos. Ya en 1995 se describió la capacidad del quitosán de interferir con la absorción de grasas. Otros estudios han confirmado que el quitosán puede captar los ácidos biliares que se sintetizan a través del colesterol en el hígado y que son vertidos al intestino, provocando una disminución de la tasa sanguínea de colesterol total y una mejoría en la relación colesterol HDL (el malo) y el colesterol total (Kang et al., 2013).

Otro ejemplo de nuevos materiales son las algas verdes marinas, que a veces tanto molestan a los bañistas. Podrían convertirse en un futuro en parte de la materia prima de los nuevos biocauchos destinados a producir neumáticos para coches (Carrasco y Pagès, 2004). La empresa italiana PireIli tiene la patente (Cataldo, et al., 2006) y parece que el nuevo material supera las pruebas estándar habituales de los cauchos que se utilizan en la fabricación de neumáticos con respecto a densidad, dureza, resistencia al rasgado, atracción, viscosidad o calentamiento.

Desde luego nos queda mucho por investigar... 
Arrieta, J.M., Arnaud-Haond, S. y Duarte, C. M. (2010). What lies underneath: conserving the oceans' genetic resources. Proceedings of the National Academy of Sciences of the United States of America, 107, pp. 18318-18324. http://dx.doi. org/10.1073/pnas.0911897107

Balseiro, P., Falcó, A., Romero, A., Dios, S., Martínez-López, A., Figueras, A., Estepa, A. y Novoa, B. (2011). Mytilus galloprovincialis myticin C: a chemotactic molecule with antiviral activity and immunoregulatory properties. PLoSOne, 6, p. e23140. http://dx.doi.org/10.1371/ journal.pone.0023140

Baranski, M., Moen, T. y Våge, D.I. (2010). Mapping of quantitative trait loci for flesh colour and growth traits in Atlantic salmon (Salmosalar). Genetics Selection Evolution, 42, p. 17. http://dx.doi. org/10.1186/1297-9686-42-17

Barboza, N.M., Medina, D. J., Budak-Alpdogan, T., Aracil, M., Jimeno, J. M., Bertino, J. R. y Banerjee, D. (2012). Plitidepsin (Aplidin) is a potent inhibitor of diffuse large cell and Burkitt lymphoma and is synergistic with rituximab. Cancer Biology and Therapy, 13, pp.114-122. http:// dx.doi.org/10.4161/cbt.13.2.18876

Carrasco, F. y Pagès, P. (2004). Kinetics of the Thermal Decomposition of Green Alga Ulva by Thermogravimetry. Journal of Applied Polymer Science, 93, pp. 1913-1922. http://dx.doi.org/10.1002/ app. 20675

Cataldo, F., Carrasco, F., Paradossi, G., Cavalieri, F., Abati, G. y Esposito, L. (2006). Procedimento e formulazioni per la preparazione di mescolanze mediante l'impiego di biomasse come ad esempio ulva e ulvano come biopolimerinaturali e loro impiego nella fabbricazione di pneumatici e di altri manufatti in gomma. Italia. Número de patente: 0001333847.

Chen, S., Zhang, C., Shao, C. et al. (2014). Whole-genome sequence of a flatfish provides insights into ZW sex chromosome evolution and adaptation to a benthic lifestyle. Nature Genetics, 46, pp. 253-260. http://dx.doi.org/10.1038/ ng. 2890

Chivian, E. y Bernstein, A. (2008). Sustaining Life. Oxford: Oxford University Press.

Davidson, W.S., Koop, B. F., Jones, S., Iturra, P., Vidal, R., Maass, A., Jonassen, I., Lien, S. y Omholt, S. (2010). Sequencing the genome of the Atlantic salmon (Salmosalar). Genome Biology, 11, p. 403.

Figueras, A. (2007). Biología y cultivo del mejillón (Mytilus galloprovincialis) en Galicia. Madrid: Consejo Superior de Investigaciones Científicas.

Figueras, A., Costa, M.M. y Novoa, B. (2012). Applications of Functional Genomics in Molluscs Aquaculture. En Saroglia, M. y Liu, J. (eds.), Functional genomics in aquaculture. West Sussex: Wiley-Blackwell, pp. 377-395. http:// dx.doi.org/10.1002/9781118350041. ch15

Ghodbane, R., Ameen, S.M., Drancourt, M. y Brunel, J.M. (2013). In vitro antimicrobial activity of squalamine derivatives against mycobacteria. Tuberculosis (Edinb), 93, pp. 565-566. http://dx.doi. org/10.1016/j.tube.2013.04.006

Gutierrez, A.P., Lubieniecki, K. P., Davidson, E. A., Lien, S., Kent, M. P., Fukui, S., Withler, R. E., Swift, B. y Davidson, W. S. (2012). Genetic mapping of quantitative trait loci (QTL) for body-weight in Atlantic salmon (Salmosalar) using a 6.5 K SNP array. Aquaculture, 358-359, pp. 61-70. http://dx.doi.org/10.1016/j. aquaculture.2012.06.017

Houston, R.D., Haley, C. S., Hamilton, A., Guy, D. R., Tinch, A. E., Taggert, J. B., McAndrew, B. J. y Bishop, S. C.(2008). Major quantitative trait loci affect resistance to infectious pancreatic necrosis in Atlantic salmon (Salmosalar). Genetics, 178, pp. 1109-1115. http://dx.doi. org/10.1534/genetics.107.082974

Iversen, E.S. (1976). Farming the edge of the sea. London: Fishing News (Books) Ltd.

Kang, N.H., Lee, W. K., Yi, B. R., Lee, H. R., Park, M. A., Park, S. K., Park, H. K. y Choi, K. L. (2013). Risk of cardiovascular disease is suppressed by dietary supplementation with protamine and chitooligosaccharide in Sprague-Dawley rats. Molecular Medicine Reports, 7, pp. 127-133.

Kijjoa, A. y Sawangwong, P. (2004). Drugs and cosmetics from the sea (review paper). Marine Drugs, 2, pp. 73-82. http:// dx.doi.org/10.3390/md202073

Ledford, H. (2013). Transgenic salmon nears approval. Nature, 497, pp. 17-18. http://dx.doi.org/10.1038/497017a

Lee, H., Scherer, N.F. y Messersmith, P.B. (2006). Single-molecule mechanics of mussel adhesion. Proceedings of the National Academy of Sciences of the United States of America, 103, pp. 12999-13003. http://dx.doi. org/10.1073/pnas.0605552103

Milne, P. H. (1972). Fish and Shellfish Farming in Coastal Waters. London: Fishing News (Books) Ltd.

Moneo, V., Serelde, B. G., Blanco-Aparicio, C., Diaz-Uriarte, R., Avilés, P., Santamaría, G., Tercero, J. C., Cuevas, C. y Carnero, A.(2014). Levels of active tyrosine kinase receptor determine the tumor response to Zalypsis. BMC Cancer, 14, p. 281. http://dx.doi.org/10.1186/14712407-14-281

Rise, M.L., von Shalburg, K. R., Brown, B. D., Mawer, M. A., Devlin, R. H., Shukin, R., Zeznik, J. A., Nelson, C., Jones, S. R., Smailus, D. E., Jones, S. J., Schein, J. E., Marra, M. A., Butterfield, Y. S., Stott, J. M., Nq, S. H., Davidson, W. S. y Koop, B. F. (2004). Development and application of a salmonid EST database and CDNA microarray: data mining and interspecific hybridization characteristics. Genome Research, 14, pp. 478-490. http:// dx.doi.org/10.1101/gr.1687304

Salazar, R., Cortés-Funes, H., Casado, E., Pardo, B., López-Martín, A., Cuadra, C., Tabernero, J., Coronado, C., García, M., Soto Matos-Pita, A., Miguel-Lillo, B., Cullel-Young, M., Iglesias Dios, J. L. y PazAres, L. (2013). Phase I study of weekly kahalalide $\mathrm{F}$ as prolonged infusion in patients with advanced solid tumors. Cancer Chemotherapy Pharmacology, 72, pp. 75-83. http://dx.doi.org/10.1007/ s00280-013-2170-5

Shimomura, O. (2009). Discovery of green fluorescent protein (GFP) (Nobel Lecture). Angewandte Chemie International Edition England, 48, pp. 55905602. http://dx.doi.org/10.1002/ anie. 200902240

Soh, L., Montazeri, M., Haznedaroqlu, B. Z., Kelly, C., Peccia, J., Eckelman, M. J. y Zimmerman, J. B. (2014). Evaluating microalgal integrated biorefinery schemes: empirical controlled growth studies and life cycle assessment. Bioresource Technology, 151, pp. 19-27. http://dx.doi. org/10.1016/j.biortech.2013.10.012

Takeuchi, T., Kawashima, T., Koyanagi, R., Gyoja, F., Tanaka, M., Ikuta, T., Shoguchi, E., Fugiwara, M., Shinzato, C., Hisata, K., Fujie, M. Usami, T., Nagai, K., Maeyama, K., Okamoto, K., Aoki, H., Ishikawa, T., Masaoka, T., Fujiwara, A., 
Endo, K., Endo, H., Nagasawa, H., Kinoshita, S., Asakawa, S., Watabe, S. y Satoh, N. (2012). Draft genome of the pearl oyster Pinctadafucata: a platform for understanding bivalve biology. DNA Research, 19, pp. 117-130. http://dx.doi. org/10.1093/dnares/dss005

Vanegas, C.H. y Bartlett, J. (2013). Green energy from marine algae: biogas pro- duction and composition from the anaerobic digestion of Irish seaweed species. Environmental Technology, 34, pp. 2277-2283. http://dx.doi.org/10.10 80/09593330.2013.765922

Wang, C.S. y Stewart, R.J. (2012). Localization of the bioadhesive precursors of the sandcastle worm, Phragmatopoma californica (Fewkes). Journal of Expe- rimental Biology, 215, pp. 351-361. http://dx.doi.org/10.1242/jeb.065011

Zhang, G., Fang, X., Guo, X. et al. (2012). The oyster genome reveals stress adaptation and complexity of shell formation. Nature, 490, pp. 49-54. http:// dx.doi.org/10.1038/nature11413 